\title{
CEPHALOSPORIN UTILIZATION EVALUATION IN A UNIVERSITY TEACHING HOSPITAL: A PROSPECTIVE STUDY
}

\author{
${ }^{*}$ Gururaja MP1, Ansu Sarah ${ }^{2}$, Laxminarayana Samaga $^{3}$, Himanshu Joshi ${ }^{1}$, Sreedharan Nair $^{4}$, CS Shastry $^{1}$ \\ ${ }^{1}$ Dept. of Pharmacology, NGSM Institute of Pharmaceutical Sciences, Mangalore \\ ${ }^{2}$ Dept. of Pharmacy Pratice, NGSM Institute of Pharmaceutical Sciences, Mangalore \\ ${ }^{3}$ Dept. of Medicine, KSHEMA, Mangalore \\ ${ }^{4}$ Dept. of Pharmacy Practice, MCOPS, Manipal \\ *Corresponding author's email: Email: gurureceptor@rediffmail.com
}

\begin{abstract}
Cephalosporins are among the commonly used class of antibiotics in hospitals and healthcare facilities world-wide and the use of newer generation of Cephalosporins have increased in the developed countries with limited number of studies investigating the drug prescribing and utilization pattern in the hospital setting. Hence the present study was carried out aiming to systematically evaluate the prescribing and sensitivity patterns of various Cephalosporins in Medicine and Surgery inpatients in a University teaching Hospital of Mangalore district, Karnataka, India. Cephalosporin utilization was evaluated in 400 inpatients of Medicine and Surgery department of a University teaching Hospital in a cross sectional prospective study for 5 months duration between June 2011-October 2011. 239(59.8\%) patients from Medicine department and 161(40.2\%) patients from Surgery department were enrolled in the present study. Patients with age group greater than or equal to 60 years received Cephalosporins maximum with $34 \%$ and $28 \%$ from Medicine and Surgery wards respectively. The median length of hospital stay was 10 days. The most widely prescribed formulation was parenterals (69\%). The third-generation Cephalosporins were widely prescribed. 131 patients (32.8\%) were prescribed Cephalosporin for surgical propylaxis and 56 patients (14\%) to prevent infections. Culture test was performed in only $66(16.5 \%)$ cases, only 43 specimens showed positive culture test. Metronidazole was the most commonly co prescribed drug. This study revealed the wider usage of Cephalosporins especially third-generation Cephalosporins. The treatment regimens implemented in most of the cases were without doing any culture sensitivity test which lead to irrational prescribing.

Key words: Cephalosporins, surgical prophylaxis, Drug utilization
\end{abstract}

\section{INTRODUCTION:}

Antibiotics are among the most common medications prescribed both in the hospital setting and the community setting. ${ }^{1}$ The overuse of antibiotics has resulted in the emergence of drug-resistant strain which is very difficult to treat, representing a major public health problem. ${ }^{2,3}$ In addition to overuse, frequency and extended duration of use of antibiotics, use of suboptimal concentrations and longer length of hospital stay are additional risk factors that have contributed to the emergence of antimicrobial resistance. Emergence of antibiotic resistant strains cause considerable impact on patient treatment and outcomes adding to the increased length of stay in the hospital, additional co-morbid conditions and increased treatment cost. ${ }^{4}$ Cephalosporins are among the commonly used class of antibiotics in hospitals and healthcare facilities worldwide and the use of newer generation of Cephalosporins have increased in the developed countries. The availability of several generations of Cephalosporin antibiotics such as Cefazolin, Cefalexin, Cefadroxil, Cefuroxime, Cefaclor, Cefotaxime, Ceftriaxone, Ceftazidime and Cefixime and their various formulations and their expanded indications have an impact on prescribing patterns in hospital and community practice. Cephalosporins range from the first generation agents which are moderate spectrum agents to broader-spectrum agents (second and third generation). The spectrum of activity broadens with each generation to include Gram negative coverage and activity against Neisseria gonorrhoeae. Many of the third and fourth generation agents can cross the blood brain barrier. Consequently, Cephalosporins are used to treat a variety of infections including septicaemia, pneumonia, meningitis, biliary-tract infections, peritonitis and UTIs. Despite the wide use of Cephalosporins, there are limited number of studies investigating the drug prescribing and utilization pattern of this antibiotic in the hospital setting. Hence the present study was undertaken.

\section{METHODS:}

This prospective cross sectional study was carried out in Medicine and Surgery inpatient Departments of K.S Hegde teaching Hospital, Deralakatte in Mangalore city. The study was designed to obtain information regarding the prescribing pattern of Cephalosporins by the medical prescribers. Duration of study period was five months (June 2011 to October 2011).

Cephalosporin prescribed during the study period was taken up for analysis. Age, sex, length of hospital stay was noted. The name, route, duration of use of Cephalosporins prescribed during the period of hospital stay were recorded. The primary indication for prescribing a Cephalosporin was documented. Results of the sensitivity 
tests of isolated microorganisms were recorded. The usages of individual and different generations of Cephalosporins were calculated.

\section{RESULTS AND DISCUSSION:}

Cephalosporins are one of the classes of antibiotics that need careful attention to ensure their rational use in this era of increased threat due to microbial resistance. However, this study revealed that this was lacking as there was excessive use of these classes of antibiotics. A total of 400 patients were prescribed Cephalosporins in Medicine $(59.8 \%)$ and Surgery $(40.2 \%)$ department. Where as in the study conducted by Pereira LM et al., most of the study patient belonged to Surgery Department. ${ }^{5}$ In the present study among patients admitted to Medicine and Surgery ward there was a preponderance of those above the age of 60 years $(31.75 \%)$. Table 1 Shows the age distribution of the patients. This factor may have influenced antibiotic prescribing as older patients were more likely to be sick and to have more serious illnesses. This was similar to that of a study conducted in Pokhara, Nepal. ${ }^{6}$ The median length of hospital stay in this study was 10 days and the average duration of patient stay in the hospital was 13.5 days (range 1day-75 days). In a previous study in the Intensive Care Unit the mean \pm SD period of hospitalization was $3.84 \pm 3.14$ days and economic constraints were a major reason for seeking an early discharge from the ICU. ${ }^{7}$ The study did not compare the length of stay among inpatients of Surgery and Medicine ward who were or were not prescribed Cephalosporins.

Among the 400 patients prescribed with Cephalosporins $37.75 \%$ were co-prescribed with other antibiotics. Similar study conducted by Shankar et al., assessed that of the 687 patients hospitalized, 203 patients were co-prescribed with other antimicrobials and $98 \%$ of patients were prescribed a single antimicrobial which was similar to that of our study. ${ }^{6}$ The most commonly prescribed antibiotic along with Cephalosporins in Medicine and Surgery ward was Metronidazole which accounted for $15.48 \%$ and $20.25 \%$ respectively. Also in a previous study Metronidazole was the mostly used antibiotic co-prescribed with Cephalosporins. $^{8}$ Metronidazole, being active against Gram-negative anaerobes, available as oral and dosage forms, rapid bacterial killing, good tissue penetration and lower expense physicians still consider it to be the 'gold standard' antibiotic against which all other antibiotics with anaerobic activity should be compared. Multiple antimicrobials usage increased due to elapse of time to diagnose the patient and to select the appropriate treatment.

Both oral and parenteral Cephalosporins were mostly prescribed in Surgery ward $(32.3 \%)$. This was due to the reason that majority of patients in Surgery ward switched over from injection to oral formulation. In Canada a route conversion program on the prescribing of antimicrobials succeeded in reducing the frequency of use of parenteral antibiotics. $^{12}$ In our hospital, patients were usually discharged once the antibiotics changed from the parenteral to the oral route. Early switchover to oral antibiotics could be more difficult for rural patients as they had difficulty in accessing medical care in case of problems. The patients were generally unwilling to stay in the hospital after injectable drugs had been stopped. The patients could be educated about the need for remaining under observation in the hospital even after the stoppage of parenteral drugs, thus a programme similar to the one in the Canadian study could be implemented in our hospital.

Among the 400 patients $34 \%$ of patients only received the test dose of Cephalosporins, usually $0.1 \mathrm{ml}$ intradermally or subcutaneously. The data collected revealed that $88.25 \%$ of total patients received third-generation Cephalosporins. The wide usage had been due to their broad spectrum of activity. This finding was less than that of a study conducted by Rachal P et al., in which total percentage of third-generation Cephalosporins was $95.85 \% .^{8}$

In our study about $98 \%$ of patients were prescribed with Cephalosporins in their trade names. This was an advantage because prescription written for the brand name would be easily dispensed. In our study Cephalosporins were prescribed for surgical prophylaxis in $33 \%$ and $40.5 \%$ for bacteriologically proven infections. Table 2: shows the indications of Cephalosporins. The most widely prescribed Cephalosporin in our study was Ceftriaxone (64.5\%). Table 3: shows Frequency of use of Cephalosporins for particular indication. Where as a study conducted by Pereira et al., showed maximum usage of Ceftriaxone with a percentage of 66 and was mainly prescribed in Surgery ward ${ }^{5}$. In our study the most frequently prescribed Cephalosporin in both Medicine and Surgery ward was Ceftriaxone with a highest percentage of 72 in Medicine ward.

In a previous study, antibiotics were used for bacteriologically proven infections in about $32 \%$ of patients. $^{6}$ In the present study third-generation Cephalosporins were prescribed to $87.79 \%$ of patients for surgical prophylaxis which was inappropriate. For surgical prophylaxis it was important to select an antibiotic with narrowest antibacterial spectrum to reduce the emergence of resistance and also broad spectrum antibiotics could be required later if patient developed serious sepsis. Therefore it was recommended that the use of third-generation Cephalosporins could be avoided in surgical prophylaxis. ${ }^{9}$ Though being expensive, Cephalosporins were used in Surgery ward to greater extent for prophylaxis due to their excellent activity against most of the bacteria causing post operative wound infection. Continued empiric use for presumed infections, prophylactic injection in surgery and empiric therapy for fever can lead to inappropriate usage of Cephalosporins. ${ }^{10}$ Hernia and appendicitis were the two common conditions in which the Cephalosporins were prescribed prophylactically.

In the present study microbiological sensitivity test was conducted in $16.5 \%$ of patients. Table 4: shows the sensitivity pattern of isolated microorganisms to Cephalosporins. Similar study was done by Palikhe et al., where specimens were taken for culture in $19.8 \%$. of cephalosporin prescribed patients. ${ }^{11}$ In the study hospital culture and sensitivity testing was not routinely employed and was done only in indicated persons as detailed. It was because of non-affordability or financial/socio-economic 
issues of the rural patients. Antimicrobial usage mainly depends on various factors like resistance, adverse effects and the cost of health care. So proper method should be implemented in order to prevent the irrational use of antimicrobials like proper understanding of therapeutic use of antimicrobials, awareness about the prevalence of various pathogens and resistance patterns to advice the proper empirical therapy.

\section{CONCLUSION:}

The present study revealed the wider usage of Cephalosporins especially third-generation Cephalosporins in Medicine and Surgery ward. Most of the patients received Cephalosporin for surgical prophylaxis(nonbacteriologically proven infection). Most widely used formulation of Cephalosporins were parenterals. The treatment regimen implemented in most of the cases were without doing any culture sensitivity test which lead to irrational prescription. Physicians should be more specific in their diagnosis despite the financial burden of culture test. An antibiotic monitoring team comprising a pharmacist, physician, medical microbiologist and infection control nurse to periodically review and evaluate the use and cost of antibiotics at this hospital to assist clinicians in optimizing clinical care of patients were needed. Antibiotic resistance is becoming a problem and formulation of a hospital antibiotic use policy is a matter of urgent concern. Implementation of antimicrobial order form will enhance the appropriateness of therapy. Although physicians were aware of problem of resistance to antibiotics and the contributory factors, their practice did not reflect measures to reduce it. The broader outcome of this study would be the potential utility of this data in designing strategies both at the level of the administrators for rational prescribing and policy decisions respectively.

Table 1: Age wise distribution of patients in Medicine and Surgery wards

\begin{tabular}{|c|c|c|c|}
\hline \multirow{2}{*}{ Age Range } & \multicolumn{2}{|c|}{ Number of patients (\%) } & \multirow{2}{*}{ Total (\%) } \\
\cline { 2 - 3 } & Medicine & Surgery & \\
\hline $18-24$ & $27(11.3)$ & $9(5.59)$ & $36(9)$ \\
\hline $24-30$ & $17(7.11)$ & $8(4.97)$ & $25(6.25)$ \\
\hline $30-36$ & $18(7.53)$ & $11(6.83)$ & $29(7.25)$ \\
\hline $36-42$ & $17(7.11)$ & $10(6.21)$ & $27(6.75)$ \\
\hline $42-48$ & $27(11.3)$ & $24(14.9)$ & $51(12.75)$ \\
\hline $48-54$ & $30(12.55)$ & $28(17.39$ & $58(14.5)$ \\
\hline $54-60$ & $22(9.20)$ & $25(15.53)$ & $47(17.25)$ \\
\hline$>=60$ & $81(33.89)$ & $46(28.57)$ & $127(31.75)$ \\
\hline
\end{tabular}

Table 2: Indications of Cephalosporins

\begin{tabular}{|l|c|c|}
\hline \multicolumn{1}{|c|}{ Indication } & Number of patients (n=400) & Percentage(\%) \\
\hline Fever & 43 & 10.75 \\
\hline Respiratory Tract Infection & 47 & 11.75 \\
\hline Gastrointestinal Infection & 32 & 8 \\
\hline Ulcer & 33 & 8.25 \\
\hline Diabetic foot ulcer & 14 & 3.5 \\
\hline Infection & 56 & 14 \\
\hline Cellulitis & 7 & 1.75 \\
\hline Surgical prophylaxis & 131 & 32.75 \\
\hline Meningitis & 8 & 2 \\
\hline UTI & 29 & 7.25 \\
\hline
\end{tabular}


Table 3: Frequency of use of Cephalosporins for particular indication

\begin{tabular}{|c|c|c|c|c|c|c|c|c|c|c|c|c|c|c|}
\hline & \multicolumn{14}{|c|}{ Number of Patients } \\
\hline Diagnosis & 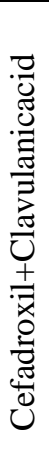 & 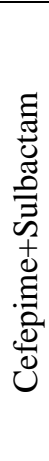 & 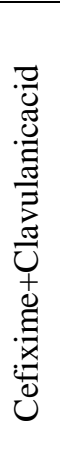 & 苞 & 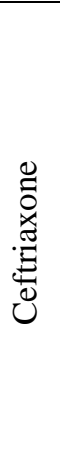 & 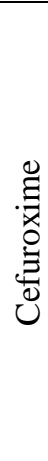 & 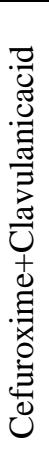 & 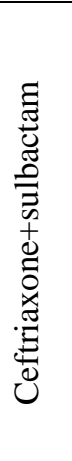 & 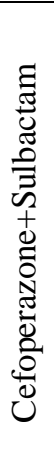 & 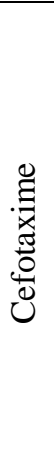 & 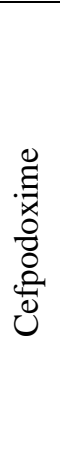 & 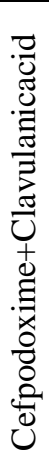 & 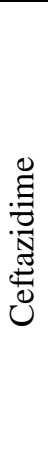 & 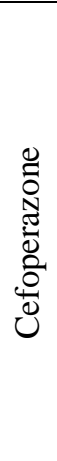 \\
\hline Infection & - & 4 & 2 & 2 & 39 & - & - & 4 & - & 2 & 2 & - & 1 & - \\
\hline Cellulitis & - & 4 & - & - & 2 & - & - & - & - & 1 & - & - & - & - \\
\hline $\begin{array}{c}\text { Surgical } \\
\text { Prophylaxis }\end{array}$ & - & 9 & 3 & 22 & 69 & 7 & - & 12 & 2 & 6 & 1 & - & - & - \\
\hline Meningitis & - & - & - & - & 6 & - & - & - & 1 & - & 1 & - & - & - \\
\hline UTI & - & - & 1 & 3 & 21 & - & - & 1 & - & 1 & 1 & - & 1 & - \\
\hline Fever & - & - & - & 1 & 38 & 1 & - & 1 & - & - & 2 & - & - & - \\
\hline RTIs & 1 & 3 & - & - & 40 & - & 1 & - & - & - & 1 & 1 & - & - \\
\hline GI infections & - & 3 & 1 & 2 & 24 & - & - & 2 & - & - & - & - & - & - \\
\hline Ulcer & - & 6 & 5 & 3 & 15 & - & - & 1 & 1 & 1 & - & - & - & 1 \\
\hline $\begin{array}{l}\text { Diabetic foot } \\
\text { ulcer }\end{array}$ & - & 6 & - & 1 & 4 & 2 & - & 1 & - & - & - & - & - & - \\
\hline
\end{tabular}

Table 4: Sensitivity pattern of organisms to Cephalosporins(S-Sensitive R-Resistant)

\begin{tabular}{|l|c|c|c|c|c|c|c|c|}
\hline \multirow{2}{*}{ Organism } & \multicolumn{2}{|c|}{ Cefepime } & \multicolumn{2}{c|}{ Ceftazidime } & \multicolumn{2}{c|}{ Ceftriaxone } & \multicolumn{2}{c|}{ Cefotaxime } \\
\cline { 2 - 9 } & $\mathbf{S}$ & $\mathbf{R}$ & $\mathbf{S}$ & $\mathbf{R}$ & $\mathbf{S}$ & $\mathbf{R}$ & $\mathbf{S}$ & $\mathbf{R}$ \\
\hline Acinetobacter & $28.6 \%$ & $42.9 \%$ & $28.6 \%$ & $57.1 \%$ & $28.6 \%$ & $42.9 \%$ & - & $14.3 \%$ \\
\hline E.coli & - & $60 \%$ & $20 \%$ & $60 \%$ & $20 \%$ & $60 \%$ & $20 \%$ & $60 \%$ \\
\hline $\begin{array}{l}\text { Gram-negative } \\
\text { bacilli }\end{array}$ & - & - & $50 \%$ & $50 \%$ & $50 \%$ & $50 \%$ & $20 \%$ & $50 \%$ \\
\hline Klebsiella spp. & $16.7 \%$ & $50 \%$ & $16.7 \%$ & $66.7 \%$ & $25 \%$ & $75 \%$ & $25 \%$ & $58.3 \%$ \\
\hline Proteus vulgaris & - & $33.3 \%$ & $66.7 \%$ & $33.3 \%$ & $66.7 \%$ & $33.3 \%$ & $66.7 \%$ & $33.3 \%$ \\
\hline $\begin{array}{l}\text { Pseudomonas } \\
\text { aeruginosa }\end{array}$ & $54.5 \%$ & $27.3 \%$ & $72.7 \%$ & $27.3 \%$ & - & $18.2 \%$ & - & - \\
\hline Enterobacter & - & $100 \%$ & - & $100 \%$ & - & $100 \%$ & - & $50 \%$ \\
\hline $\begin{array}{l}\text { Proteus } \\
\text { mirabilis spp. }\end{array}$ & - & $100 \%$ & - & $100 \%$ & - & $100 \%$ & - & - \\
\hline
\end{tabular}

CONFLICTS OF INTEREST: NIL

\section{ACKNOWLEDGMENTS:}

The Authors are thankful to NITTE University, Mangalore, Karnataka for providing necessary facilities to carry out this study. 


\section{REFERENCES:}

1. Meer JW, Gyssens IC, Quality of antimicrobial drug prescription in hospital, Clin Microbiol Infect., 2001,7( 6), 12-5.

2. Goosens H, Antibiotic consumption and link to resistance, Clin Microbiol Infect. , 2009,15 (3), 12-5.

3. Austin DJ, Kristinsson KG, Anderson RM, The relationship between the volume of antimicrobial consumption in human communities and the frequency of resistance, Proc Natl Acad Sci USA, 1999, 96(3), 1152-6.

4. Shorr AF, Epidemiology and economic impact of meticillinresistant Staphylococcus aureus: review and analysis of the literature, Pharmacoeconomics, 2007, 25(9), 751-68.

5. Pereira LM, Phillips M, Ramlal H, Teemul K, Prabhakar P, Third generation cephalosporin use in a tertiary hospital in Port of Spain, Trinidad: need for an antibiotic policy, BMC Infect Dis., 2004, 4(59), 1-7.

6. Shankar PR, Partha P, Shenoy NK, Easow JM, Narayanan K, Prescribing patterns of antibiotics and sensitivity patterns of common microorganisms in the Internal Medicine ward of a teaching hospital in western Nepal: a prospective study, Ann Clin Microbiol Antimicrob, 2003, 2(7), 1-9.
7. Shankar PR, Partha P, Shenoy N, Brahmadathan KN, Investigation of antimicrobial use pattern in the intensive care unit of a teaching hospital in western Nepal, Am J Infect Control, 2003, 31(7), 410-4.

8. Paul R, Sadiq MS, Kannan, A Prospective Study on Usage of Cephalosporins in Hospitalized Patients, IJPI's Journal of Hospital and Clinical Pharmacy, 2011, 1(5), 1-8.

9. Munckhof W, Antibiotics for surgical prophylaxis, Aust Prescr., $2005,28,38-40$

10. Lee H, Jung D, Yeom JS, Son JS, Jung SI, Kim YS, et al., Evaluation of Ceftriaxone utilization at Multicenter Study, Korean J Intern Med., 2009, 24(4), 374-80.

11. Palikhe N, Prescribing pattern of Antibiotics in Paediatric Hospital of Kathmandu valley, Kathmandu University Medical Journal, 2004, 2(1), 6-12.

12. Zamin HT, Pitre MM, Conly JM, Development of an intravenous-to-oral route conversion program for antimicrobial therapy at a Canadian tertiary health care facility, Ann $\begin{array}{lllr}\text { Pharmacother., } & 1997, & 31, & 564-70 .\end{array}$ 